\title{
Diagnóstico de las afectaciones socio-ambientales por el ruido en el procesamiento de cacao de la corporación CAORO en el sitio Río Negro
}

\section{Diagnosis of socio-environmental effects due to noise in cocoa processing of the CAORO corporation at Río Negro site}

\author{
Yuri Patricio Espinoza Aguilar ${ }^{1 *}$, Alex Dumany Luna Florin ${ }^{1}$ y Maria Belén Buele Calderón ${ }^{1}$ \\ ${ }^{1}$ Universidad Técnica de Machala \\ *yespinoza@utmachala.edu.ec
}

DOI: https://doi.org/10.26871/killkana_social.v2i3.321

\begin{abstract}
Resumen
En el sector Río Negro perteneciente a la parroquia La Victoria del cantón Santa Rosa, la corporación CAORO (Corporación Agroexportadora El Oro) ha venido desarrollando la actividad de la compra de cacao donde se realiza la fermentación y secado del mismo para la comercialización a nivel nacional y posterior exportación, esta actividad presenta impactos sociales y ambientales los cuales se darán a conocer en la presente investigación. Entre los impactos sociales se encontró que los trabajadores están expuestos por un periodo de tiempo extenso al ruido con decibeles que superan los límites permisibles establecidos en el Texto Unificado de Legislación Secundaria Medio Ambiental, lo que a futuro le puede causar un riesgo a la salud como nerviosismo y sordera. En los impactos ambientales se observó que el ruido afecta a las aves terrestres como Furnarius cinnamomeus, Dives warszewiczi, Ara chloroptera, Eastern bluebird, Columba livia, Turdus merula, del sector causando que se perturben en la búsqueda de alimentos por estar siempre alerta a los depredadores, también algunas aves modificaban su comportamiento natural al trinar en este entorno ruidoso. Las vibraciones generadas por la maquinaria utilizada tienen efectos adversos en la salud del trabajador que habita en la corporación, causando espondilitis, calcificación de discos e incluso menor habilidad manual. En la fauna las vibraciones causan daños mecánicos al aparato digestivo y sistemas respiratorios, además desorientación puesto que cada animal tiene una vibración específica de energía que los ayuda a comunicarse entre sí.
\end{abstract}

Palabras clave: Secado, cacao, ruido, vibraciones, impactos.

\begin{abstract}
In the Rio Negro sector belonging to the La Victoria parish of Santa Rosa, the corporation CAORO (Agroexport Corporation El Oro) has been developing the activity of the purchase of cocoa where the fermentation and drying of the same is carried out for the commercialization at the national level And subsequent export, this activity has social and environmental impacts which will be announced in the present investigation. Among the social impacts were found that workers are exposed for an extended period of time to noise with decibels that exceed the permissible limits established in the Unified Text of Secondary Environmental Legislation, which in the future can cause a health risk as Nervousness and deafness. In environmental impacts, it was observed that the noise affects terrestrial birds such as Furnarius cinnamomeus, Dives warszewiczi, Ara chloroptera, Eastern bluebird, Columba livia, Turdus merula, of the sector causing them to be disturbed in the search of food for being always alert to Predators, some birds also modified their natural behavior by treading in this noisy environment. The vibrations generated by the machinery used have adverse effects on the health of the worker who lives in the corporation, causing spondilitis, disc calcification and even less manual ability. In the wild the vibrations cause mechanical damages to the digestive system and respiratory systems, besides disorientation since each animal has a specific vibration of energy that helps them to communicate with each other.
\end{abstract}

Key words: Drying, cocoa, noise, vibrations, impacts.

\section{Introducción}

La corporación CAORO (Corporación Agroexportadora El Oro), fue fundada el 5 de julio de 1999, con un área de $100 \mathrm{~m} 2$ sin fines de lucro. Cuenta con 20 socios, y su directiva está conformada por la presidenta Lic. Carmen Alejandrina Calderón Torres, el gerente Sr. Felipe Jácome y la secretaria Ing. Anabel Ramón. Esta corporación se encuentra ubicada en la parroquia La Victoria del cantón 
Santa Rosa, sector Rio Negro, a 25 minutos del cantón Santa Rosa y a 45 minutos del cantón Machala, el sector tiene una temperatura promedio de entre 20 a $23^{\circ} \mathrm{C}$, con una altura de $30 \mathrm{msnm}$. (?, ?)

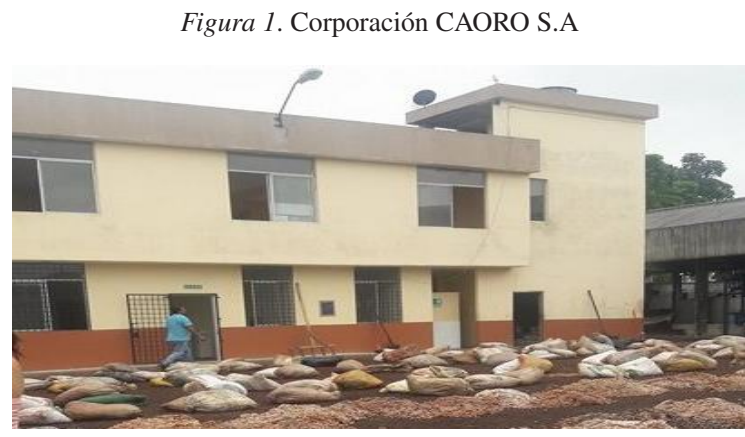

Fuente: Los autores

Figura 2. Instalaciones de la corporación

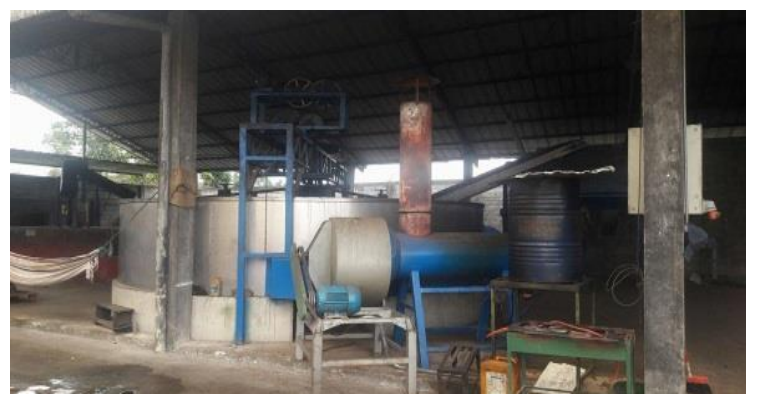

Fuente: Los autores

La corporación CAORO (Corporación Agroexportadora El Oro) se dedican a la compra de cacao para realizar el proceso de fermentación y secado utilizando una maquina secadora manual con capacidad de 60 qq y dos secadoras automáticas con capacidad de 30 qq, luego venden a un costo de $\$ 70.00$ por quintal a la empresa AGROARRIBA de la ciudad de Guayaquil aproximadamente 150 qq por semana. Para este proceso la corporación cuenta con cuatro trabajadores eventuales y uno fijo.

Con esta investigación se busca diagnosticar las afectaciones que se están produciendo en la salud de los trabajadores, población aledaña y el medio ambiente por el ruido y vibraciones de las maquinas utilizadas en la corporación CAORO (Corporación Agroexportadora El Oro), entre ellas disminución de la capacidad auditiva, dificultades para conciliar el sueño, estrés y alteración de la comunicación oral (?, ?), en la fauna afecta principalmente a las aves más representativas como el Furnarius cinnamomeus, Dives warszewiczi, Ara chloroptera, Eastern bluebird, Columba livia, Turdus merula, provocando desorientación, migración y alejamiento de los hábitats naturales y estrés al resto de las especies (?, ?).

La exposición prolongada al ruido, puede causar múltiples efectos a la salud entre los que podemos citar: respiratorios, cardiovasculares, digestivos, visuales, endocrinos y sistema nervioso. Se conoce que el ruido puede afectar adversamente a la lectura, la atención, la resolución de problemas y la memoria. (?, ?)

Existen otras molestias que afectan a la salud humana tales como: irritabilidad, tensión nerviosa, lo cual origina fatiga precoz, puede causar molestia, accidentes y favorecer el ausentismo, disminución de la resistencia eléctrica de la piel, reducción de la actividad gástrica, aceleración temporal del ritmo respiratorio y cardíaco con aumento de la presión arterial, si es extremadamente excesivo, pueden producirse sensaciones de nistagmo y oscilaciones involuntarias de los glóbulos oculares, hay variaciones en el sueño y disminución de la capacidad de visión para los colores y las formas. (?, ?)

Para el diagnóstico de las afectaciones se utiliza las técnicas de investigación como la observación del lugar, para la medición del ruido se empleará un sonómetro, donde se identificará si los datos obtenidos están dentro de los límites máximos permisibles, para el ruido y vibraciones, establecidos en el TULSMA, una entrevista a los dueños y trabajadores, y una encuesta a los pobladores aledaños.

$\mathrm{Al}$ diagnosticar las afectaciones se busca dar a conocer los resultados y así implementar un programa de seguridad que disminuya los efectos dañinos causados por el ruido y vibraciones, en los trabajadores se sugieren acciones como, utilizar protección para sus oídos, ya sea con tapones u orejeras. También existen los cascos anteridios con similitud a las orejeras, pero con doble función que es la de proteger la cabeza. Pero los más usados son las orejeras, las cuales si son bien seleccionadas son más eficientes que los tapones y los cascos. (?, ?). Con respecto a la población aledaña y medio ambiente se sugiere en las maquinarias, reemplazar, de ser posible las piezas de metal por piezas de plástico las cuales son más silenciosas, además impedir el choque entre las piezas de la máquina; y en cuanto a las vibraciones se propone colocar las maquinas sobre colchonetas $\mathrm{u}$ otros materiales que puedan amortiguar la intensidad de las agitaciones.

Para lograr el uso correcto de los protectores auditivos en los trabajadores, y el condicionamiento de las maquinas es necesario contar con la disposición de los socios de la Corporación para implementar estas estrategias, de tal manera que se realice controles periódicos para la verificación de las medidas adoptadas.

\section{Metodología}

La investigación se la realizo in situ utilizando el método de investigación cuantitativo para lo cual se empleó un sonómetro de marca MICROCLIPXT, fue calibrado el 2 de junio de 2014, que es un instrumento que mide la intensidad del ruido, para ello se tomó las medidas cuando las maquinas secadoras estaban en funcionamiento, en el horario de 10:00 am a 11:00 am con la finalidad de obtener datos confiables, luego se hizo una comparación con los límites máximos permisibles para ruido establecidos en el TULSMA (Texto Unificado de Legislación Secundaria 
Medio Ambiental) y dar a conocer los resultados a la Corporación CAORO (Corporación Agroexportadora El Oro) para que tomen medidas que disminuyan los riesgo.

El sonómetro empleado tiene las siguientes características:

\section{MODELO: MC2 - XWHM - Y NA}

- Mide ruidos estables, realiza una lectura directa y puntual de la presión sonora.

- Posee un dosímetro que determina el nivel del ruido.

- Micrófono de Frecuencia; a lo largo de su rango operativo, desde la frecuencia más baja a la más alta.

- Contiene amplificador; Amplifica la señal del micrófono lo suficiente para poder medir los niveles de presión sonora más bajos.

- Filtros de frecuencia: Permiten incorporar a la medida las curvas de ponderación $\mathrm{A}, \mathrm{B}, \mathrm{C}$ o $\mathrm{D}$, según el objetivo de la medición.

- Rectificador e integrador: Antes de entrar al rectificador, la señal se amplifica de nuevo.

Para la toma de niveles de ruido, se tomó una muestra por cada instalación de la corporación, las mismas que son la entrada y salida de la corporación, en la oficina y vivienda, en la bodega, en el lugar donde funciona la maquinaria y por último en el lugar donde se deja el cacao. Las muestras se tomaron a una distancia de 10 a 20 aproximadamente entre puntos.

Además, se empleó el método cualitativo como la entrevista a los trabajadores de la Corporación CAORO para determinar el tiempo de la jornada laboral que tienen y a los socios para verificar si conocen las exposiciones que tiene sus trabajadores a los riesgos por el ruido. Se aplicó una encuesta a los pobladores aledaños a la corporación con la finalidad de determinar si presentan molestias a causa del ruido y se les preguntara si han visto cambios con respecto a la presencia de aves y demás especies.

Se realizó una observación en la empresa y a sus alrededores para valorar la información tomada por parte de trabajadores, socios y pobladores y desde un punto subjetivo aportar manifestaciones que no hayan sido tomadas en cuenta con respecto a los riesgos socio-ambientales por parte de los entrevistados y encuestados.

\section{Resultados}

3.1 Resultados de la entrevista aplicada a los socios de la Coorporacion CAORO S.A

\subsubsection{Motivo de implementar máquinas para el proceso} de secado.

El motivo por el que se implementaron estas maquinarias fue por el rápido secado del producto además que abarca más cantidad que el tendal y en poco tiempo ya está seca la almendra.

\subsubsection{Contaminación por el funcionamiento de las máqui- nas.}

El socio se encuentra consiente del daño que podría causar a la salud del trabajador al no contar con el equipo de seguridad adecuado, es por eso que estos trabajadores cuentan con un debido equipo de protección, pero es responsabilidad de ellos algún daño causado por que no le dan el uso respectivo.

\subsubsection{Indicaciones por parte de profesionales al personal} que labora sobre normas de seguridad.

El personal que labora en esta empresa si ha recibido las debidas capacitaciones debido a los altos niveles de ruidos a los que están expuestos, esto lo ha llevado a cabo el personal del MAGAP y MAE.

\subsubsection{Asesoramiento recibido.}

Se ha recibido asesoramientos solo para los socios en lo que es la producción de cacao, tratamiento contra las plagas, mantenimiento de su finca, manejo de cosecha y parámetros con los que debe cumplir cada socio para su comercialización.

\subsubsection{Apertura del producto al mercado.}

La apertura de este producto al mercado ha sido exitosa en su actividad comercial sus ventas han tenido un aumento progresivo, vendiendo a grandes empresas exportadoras de cacao que tienen sus operaciones en la provincia del Guayas.

\subsubsection{Resultados del trabajo}

Si se ha tenido buenos resultados desde que inició sus actividades hasta la actualidad tanto para la empresa como para los pequeños productores de la zona ya que este producto constituye ingresos seguros.

\subsection{Resultados de la entrevista aplicada a los trabajado- res de la corporación CAORO S.A}

\subsubsection{Horario de trabajo.}

El horario de los trabajadores es de 08:00 am a 12:00 pm una hora de almuerzo y de ahí retornan a sus actividades a las 13:00 pm hasta las 18:00 pm, los trabajadores almuerzan en la empresa mismo, por lo que están expuestos al ruido constantes generados por las máquinas, uno de ellos el señor Enrique Robertino Carchipulla Ayala de 50 años de edad, quien vive ahí junto a su esposa y sus dos hojas de 7 y 12 años de edad, ellos se encuentra expuestos al ruido todo el día y en época de cosecha de cacao donde requiere más secado puede ser toda la noche, ya que las maquinas quedan encendidas hasta el siguiente día.

\subsubsection{Capacitación recibida y equipo de protección.}

Los trabajadores nos supieron manifestar que no han recibido ninguna charla acerca del cuidado que se debe tener al usar este tipo de maquinaria. Sin embargo, nos afirmaron que, si tienen equipos de protección ante el ruido generado, como cascos y tampones para los oídos, pero no los usan porque alegan que les incomoda y que están ya acostumbrados al ruido constante. 


\subsubsection{Problemas en la salud.}

Con respecto a los problemas en la salud generados por el exceso de ruido y vibraciones del lugar, los trabajadores manifestaron que al principio sufrieron de dolores de cabeza y estrés, pero que se ha ido atenuando al paso del tiempo.

\subsection{Resultados de observacion directa}

Durante la entrevista se tomaron en cuenta algunos datos curiosos entre ellos es que el señor que habita ahí ha adoptado cierta conducta del habla, con ruido eleva la voz para hablar por las interrupciones del mismo, pero al encontrarse en un área abierta donde la presencia del ruido es mínima el sigue manteniendo el mismo nivel de voz que al estar con ruido. El señor que habita ahí es uno de los más afectados por la constante exposición al ruido lo que le provoca estrés y dolores de cabeza, pero nos comenta que ellos mastican una especie conocida como albaca blanca, esta planta desde el punto de vista de ellos les quita el estrés y los malestares presentados, pero este análisis no ha sido comprobado así que se desconoce su validez.

Falta de señaléticas: Durante el recorrido hecho se observa que en la corporación no existen las debidas señaléticas sobre el uso de los equipos de protección para los trabajadores.

Presencia de olores: debido al proceso de fermentación los olores generados no son agradables al olfato humano, y los trabajadores tampoco usan las mascarillas correspondientes que disminuyan estos olores.

Ausencia de aves: se preguntó sobre si han presenciado la ausencia de aves debido al ruido, lo que comentaron es que unen inicio se alejaron, pero con el paso del tiempo se han ido acercando y que consideran que se adaptaron al ruido debido a que en el lugar cuenta con alimentación debido a los residuos de las almendras del cacao.

3.4 Resultado de la encuesta aplicada a los pobladores del sitio Rio Negro

¿Cuánto tiempo lleva usted viviendo cerca de la empresa?

Tabla 1

\begin{tabular}{ccc}
\hline Variable & Frecuencia & Porcentaje \\
\hline 1 año & 2 & $20 \%$ \\
3 años & 2 & $20 \%$ \\
Más de 3 años & 6 & $60 \%$ \\
\hline Total & 10 & $100 \%$
\end{tabular}

Fuente: Encuesta directa

Elaboración: Los autores

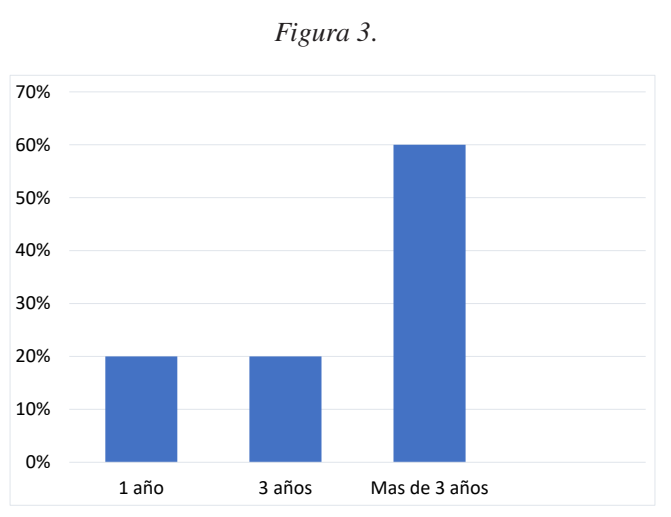

Fuente: Tabla 1

Elaboración: Los autores

Dentro de las diez familias encuestadas tenemos que el $60 \%$ tienen más de 3 años siendo aledaños a la empresa, mientras que en el rango de 3 años y de 1 año un porcentaje paralelo del $20 \%$ viviendo cerca de la corporación.

¿Le afecta el ruido generado por la maquinaria de la corporación CAORO?

Tabla 2

\begin{tabular}{ccc}
\hline Variable & Frecuencia & Porcentaje \\
\hline $\mathrm{Si}$ & 2 & $20 \%$ \\
No & 8 & $80 \%$ \\
\hline Total & 10 & $100 \%$
\end{tabular}

Fuente: Encuesta directa

Elaboración: Los autores

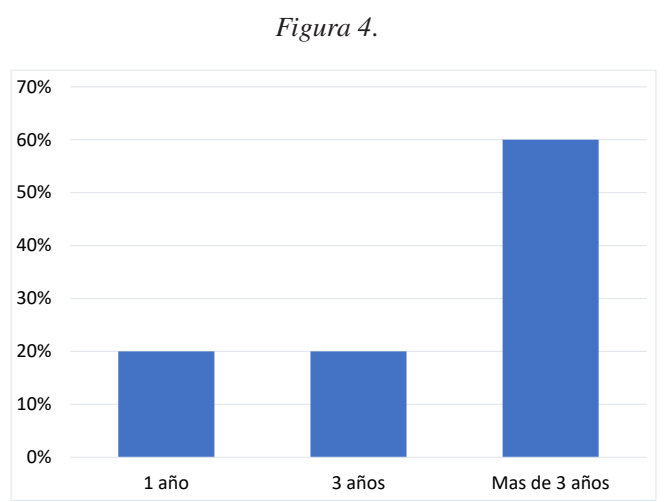

Fuente: Tabla 2

Elaboración: Los autores

Dentro de las 10 familias tenemos que el $80 \%$ no les afecta el ruido debido a que alegan que el ruido percibido es mínimo, sin embargo, un $20 \%$ alegan que si le afecta el ruido generado por las máquinas de la corporación.

¿Qué tanto le afecta el ruido generado por la maquinaria de la corporación CAORO? 
Tabla 3

\begin{tabular}{ccc}
\hline Variable & Frecuencia & Porcentaje \\
\hline Mucho & 0 & $0 \%$ \\
Poco & 2 & $20 \%$ \\
Nada & 8 & $80 \%$ \\
\hline Total & 10 & $100 \%$
\end{tabular}

Fuente: Encuesta directa

Elaboración: Los autores

Figura 5 .

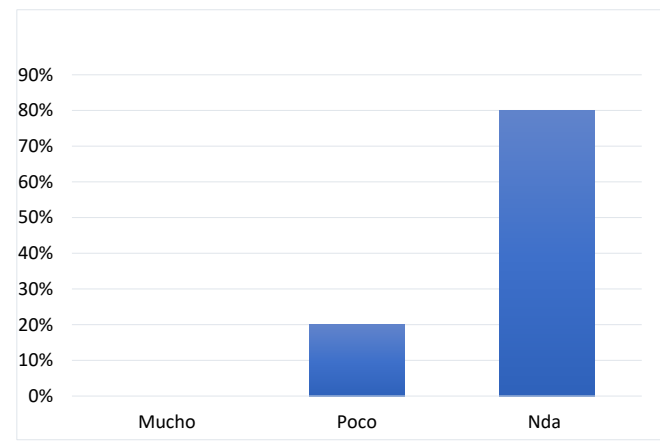

Fuente: Tabla 3

Elaboración: Los autores

De las 10 familias encuestadas, el $80 \%$ de ellas manifestaron que el ruido generado por las maquinarias de la corporación CAORO S.A no les afecta nada y que ya se han acostumbrado a eso, mientras que el $20 \%$ dijo que si les afecta un poco.

¿Qué problemas en la salud ha sufrido por el ruido generado?

\begin{tabular}{ccc} 
& Tabla 4 \\
\hline Variable & Frecuencia & Porcentaje \\
\hline $\begin{array}{c}\text { Disminución } \\
\text { auditiva }\end{array}$ & 0 & $0 \%$ \\
$\begin{array}{c}\text { Dolores de } \\
\text { cabeza }\end{array}$ & 1 & $10 \%$ \\
Estrés & 1 & $10 \%$ \\
Ninguna & 8 & $80 \%$ \\
\hline Total & 10 & $100 \%$
\end{tabular}

Fuente: Encuesta directa

Elaboración: Los autores
Figura 6.

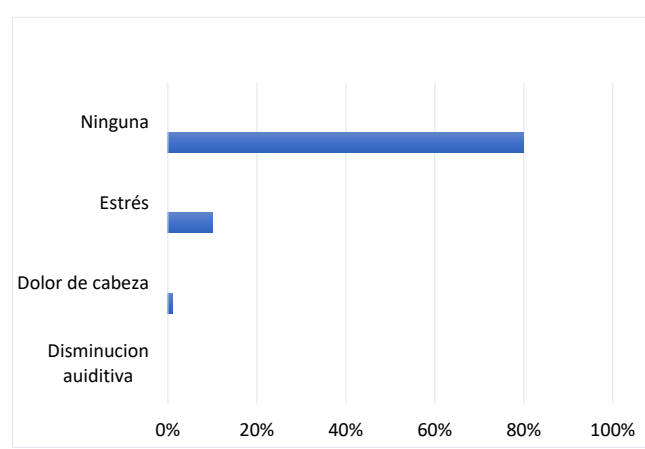

Fuente: Tabla 4

Elaboración: Los autores

De todas las familias encuestadas el $80 \%$ supo manifestar que hasta el momento no han sufrido ningún tipo de enfermedades por el ruido de las maquinarias, el $10 \%$ ha sufrido estrés y el otro $10 \%$ ha sufrido dolores de cabeza a causa del ruido generado en la corporación CAORO.

¿Siente usted las vibraciones generadas por la maquinaria de la corporación CAORO?

Tabla 5

\begin{tabular}{ccc}
\hline Variable & Frecuencia & Porcentaje \\
\hline $\mathrm{Si}$ & 0 & $0 \%$ \\
$\mathrm{No}$ & 10 & $100 \%$ \\
\hline Total & 10 & $100 \%$
\end{tabular}

Fuente: Encuesta directa

Elaboración: Los autores

Figura 7.

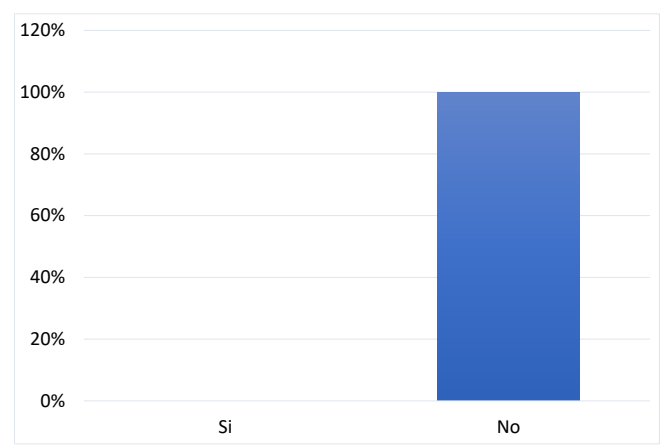

Fuente: Tabla 5

Elaboración: Los autores

De todas las familias encuestadas el $100 \%$ supo manifestar que no sienten las vibraciones que se generan durante el proceso de funcionamiento de la corporación CAORO, esto se debe a que las viviendas no están cercanas a la corporación.

¿Qué tanto le afecta las vibraciones generadas por la maquinaria de la corporación CAORO? 
Tabla 6

\begin{tabular}{ccc}
\hline Variable & Frecuencia & Porcentaje \\
\hline Mucho & 0 & $0 \%$ \\
Poco & 0 & $0 \%$ \\
Nada & 10 & $100 \%$ \\
\hline Total & 10 & $100 \%$
\end{tabular}

Fuente: Encuesta directa

Elaboración: Los autores

Figura 8.

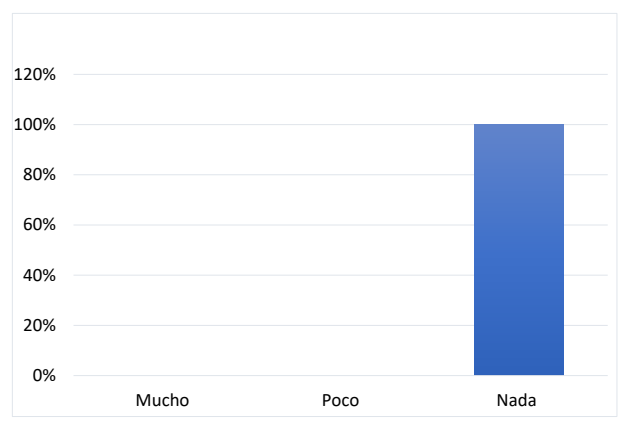

Fuente: Tabla 6

Elaboración: Los autores

De todas las familias encuestadas el $100 \%$ supo manifestar que no presentan afectaciones de ningún grado a causa de las vibraciones que se generan durante el proceso de funcionamiento de la corporación CAORO, debido a que las residencias no están cercanas a la corporación.

¿Qué problemas en la salud ha sufrido por las vibraciones generados?

Tabla 7

\begin{tabular}{ccc}
\hline Variable & Frecuencia & Porcentaje \\
\hline Problemas en la columna & 0 & $0 \%$ \\
Dificultad para dormir & 0 & $0 \%$ \\
Estrés & 0 & $0 \%$ \\
Ninguna & 10 & $100 \%$ \\
\hline Total & 10 & $100 \%$
\end{tabular}

Fuente: Encuesta directa

Elaboración: Los autores

Figura 9.

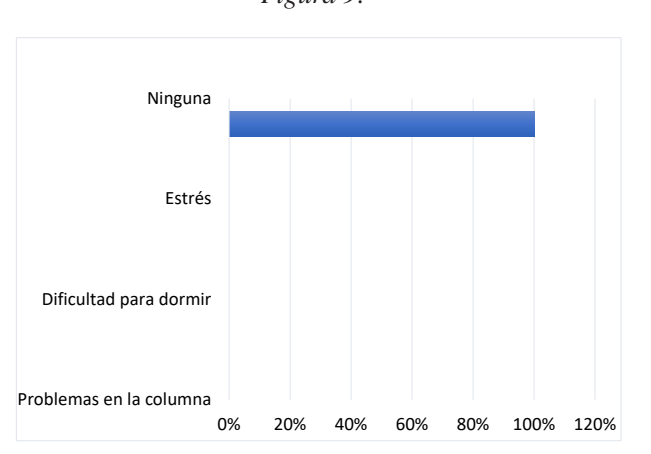

Fuente: Tabla 7

Elaboración: Los autores
Como se observa (tabla 7 y grafico 9) los pobladores no han tenido problemas en la salud a causa de las vibraciones, esto se da porque estas no son tan intensas como para llegar a las viviendas de los pobladores, por ello los mismos no tienen ningún inconveniente con el funcionamiento de la corporación CAORO S.A.

\subsection{Resultados del sonómetro}

Tabla 8 Muestras obtenidas y límites permisibles del TULSMA

\begin{tabular}{|l|c|c|}
\hline \multicolumn{1}{|c|}{ Área } & Niveles (dB) & $\begin{array}{c}\text { Límites } \\
\text { permisibles } \\
\text { establecidos en } \\
\text { el TULSMA } \\
\text { (dB) }\end{array}$ \\
\cline { 1 - 2 } Entrada de la corporación & Hi 75 & \multirow{2}{*}{70} \\
\cline { 1 - 2 } Oficina y vivienda & Hi 71 & \multirow{2}{*}{ Hi 87.3 } \\
\cline { 1 - 2 } Bodega & Hi 98 & \\
\hline Maquinas secadoras manuales (2) & Hi 97.1 \\
\cline { 1 - 2 } Maquina secadora automática & Hi 82.8 & \\
\cline { 1 - 2 } Salida de la corporación & \multicolumn{2}{|c|}{} \\
\hline
\end{tabular}

Fuente: Corporación CAORO S.A. y (?, ?)

Elaboración: Los autores

Los datos tomados en los diferentes puntos de la corporación CAORO S.A, con el sonómetro debidamente certificado son comparados con el nivel establecido en el TULSMA para zonas industriales, para lo cual se realiza el siguiente análisis:

Entrada a la corporación. - Esta área que se encuentra a 20 metros del punto de origen de ruido presenta un nivel de $75 \mathrm{~dB}$, pues está aislado del lugar de las máquinas.

Oficina y vivienda. - Los niveles de ruidos obtenidos son de $71 \mathrm{~dB}$, siendo el área con menor contaminación acústica, la vivienda presenta ventanas que actúan como aislamiento para que el ruido generado no entre con una intensidad elevada. Sin embargo, el 71dB sobrepasa el límite establecido a zonas industriales y en ese lugar no debe existir una residencia o si existe estar debidamente equipada ya que el límite mínimo para una residencia es de $50 \mathrm{~dB}$.

Bodega. Presenta niveles de $87.3 \mathrm{~dB}$ ya que está ubicada a lado del área donde funcionan las máquinas que realizan el proceso de secado del cacao.

Maquinas secadoras manuales (2). - En conjunto las dos máquinas generan un ruido de $98 \mathrm{~dB}$, siendo elevado para los parámetros establecidos en el TULSMA, sin embargo, se recalca que las máquinas que funcionan son 3 por lo que el nivel de ruido en ocasiones aumenta.

Maquina secadora automática. - Esta máquina, a pesar de ser la única automática es la que más genera ruido en la corporación con un nivel de $97.1 \mathrm{~dB}$, excediendo en gran medida al límite máximo permisible establecido por el TULSMA. 
Figura 10. Maquinas secadoras manuales

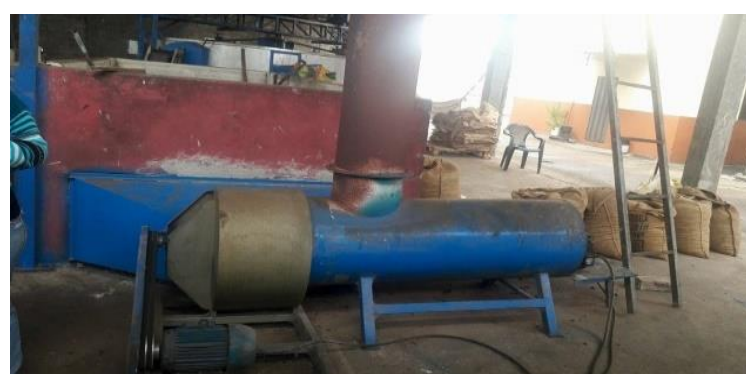

Fuente: Autores

Figura 11. Maquinas secadoras automática

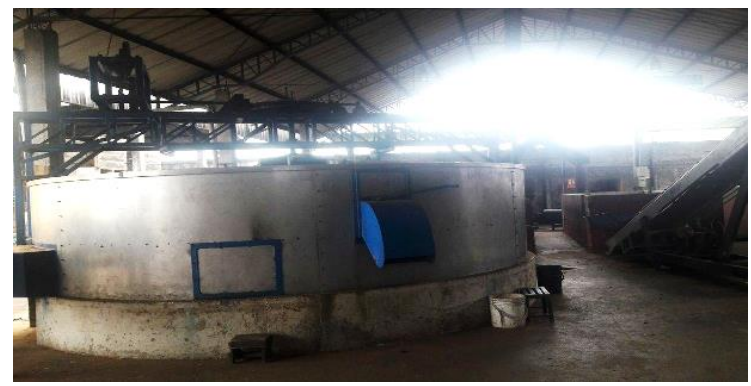

Fuente: Autores

Salida de la corporación. - Esta área tiene un nivel de ruido de $82.8 \mathrm{~dB}$, ya que se encuentra ubicada relativamente cerca del área donde se da el proceso de secado.

\section{Conclusiones}

En base a los resultados obtenidos se puede evidenciar que los trabajadores de la empresa CAORO en un principio si sufrieron algún tipo de enfermedad por el ruido, pero actualmente ya no porque ya se han adaptado al mismo, el cual es generado por las maquinarias que utilizan en la empresa, también se puede evidenciar que ellos no utilizan el equipo adecuado para laborar lo que más adelante podría provocarles algún tipo de afectación a su salud.

Se puede ver también que esta empresa a pesar de no contar con un equipo de última tecnología hasta el momento ha tenido buenos resultados ya que la acogida de su producto ha ido aumentando en el mercado comercial.

Otro punto que se pudo evidenciar es que el ruido generado por las maquinarias no es demasiado elevado por lo que los moradores del lugar manifestaron que a ellos no les ha afectado en nada la presencia de esa empresa ya que el ruido que se escucha es mínimo y ya se han adaptado a él.

\section{Referencias Bibliográficas}

Recibido: 30 de junio de 2018

Aceptado: 3 de septiembre de 2018

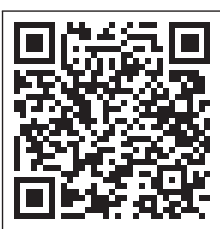


